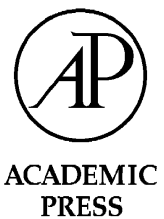

Available online at www.sciencedirect.com

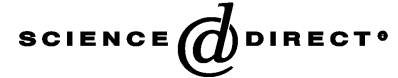

Journal of Sound and Vibration 261 (2003) 185-191

\title{
A note on the acoustic insulation between two-dimensional acoustic spaces at low frequencies
}

\author{
P. Santos*, A. Tadeu \\ Department of Civil Engineering, Faculty of Sciences and Technology, University of Coimbra, Polo II-Pinhal de Marrocos, \\ P-3030-290 Coimbra, Portugal
}

Received 23 May 2002; accepted 7 August 2002

\section{Introduction}

The acoustic insulation provided by separating walls has been studied for many years, with the first publications dating from the early part of the 20th century [1]. Since then, different analytical and numerical models, for predicting the sound transmission via single and multi-panel walls, have been proposed. However, given the large number of variables of this problem and the uncertainties involved, laboratory tests have been devised to measure the insulation provided by separating elements. Among the various experimental testing methods, the most commonly used technique is the so-called two-room method [2-5].

It has been shown that, at low frequencies, the sound reduction index is highly dependent upon parameters such as the size of the testing chambers, sound source location and rooms' surface absorption conditions [6-9], making it difficult to extrapolate test results to real-world situations. Indeed, the sound insulation provided by a wall is highly dependent on the vibration modes excited in the rooms, and on the vibration of the separating wall.

The present work assesses the influence of the parallelism between the boundaries of the acoustic spaces and the importance of the rooms' surface conditions on the characterization of the sound insulation offered by a wall separating two contiguous two-dimensional rooms. It makes use of a boundary element method (BEM) model, formulated in the frequency domain, previously developed by the authors [10], which takes into account the interaction, between the air and the solid walls. The acoustic insulation provided by walls of infinite extent is used as a reference ("half-space room"). These solutions are obtained analytically, taking also the full coupling between the air and the solid into account [11].

The formulation of the BEM method and the derivation of the analytical solution are not presented here, since they have been already given by the authors [10,11]. A survey of the state of the art more relevant to the present work can also be found in Santos et al. [10]. The remainder of

\footnotetext{
*Corresponding author. Tel.: +351-239-797201; fax: +351-239-797190.

E-mail address: pfsantos@dec.uc.pt (P. Santos).
} 
this paper presents only a selection of simulations, for different rooms' cross-sections and materials to illustrate the main findings.

\section{Applications}

The model used consists of two adjacent two-dimensional acoustic spaces separated by a vertical single wall, $0.20 \mathrm{~m}$ thick (two-room model). Fig. 1 displays the geometry of the model, which assumes that the ceiling of the acoustic spaces is horizontal while the back walls and floor may be inclined $\theta$ degrees, in relation to the vertical $(Y)$ and horizontal $(X)$ directions, respectively. Simulations for $\theta=0^{\circ}, 5^{\circ}$, and $15^{\circ}$ are presented next.

The lining of the acoustic spaces is assumed to be thick and to have material properties similar to those of the surrounding elastic medium. The internal material loss is considered using a complex Young's modulus and complex Lamé constants. The Young's modulus is computed as $E=E_{r}(1+\mathrm{i} \eta)$, where $E_{r}$ corresponds to the classic modulus and $\eta$ is the loss factor. The complex Lamé constants are written in the same form as the Young's modulus.

To assess the importance of the rooms' surface conditions, the mechanical material properties [12] of the concrete, ceramic and cork have been ascribed to the surrounding elastic medium as listed in Table 1.

The different models were subjected to the incidence of a cylindrical linear wave source, positioned in the source room as illustrated in Fig. 1. The calculations were performed for a frequency range from 1.0 to $600 \mathrm{~Hz}$, with a frequency increment of $1.0 \mathrm{~Hz}$. The acoustic pressure wave field was computed along a grid of receivers placed in the two rooms, equally spaced, $0.25 \mathrm{~m}$ apart, along the vertical and horizontal directions, as illustrated in Fig. 1.

The use of the BEM model allows the discretization to be limited to the boundaries of the acoustic spaces. These boundaries are modelled with a number of boundary elements that increases with the frequency of excitation of the dynamic source. The length of the elements is defined by the acoustic wavelength divided by 10 . Given the small distance between the two faces of the separating wall, the length of boundary elements modelling the wall follows the above relation, but must also be at least 8 times less than its thickness.

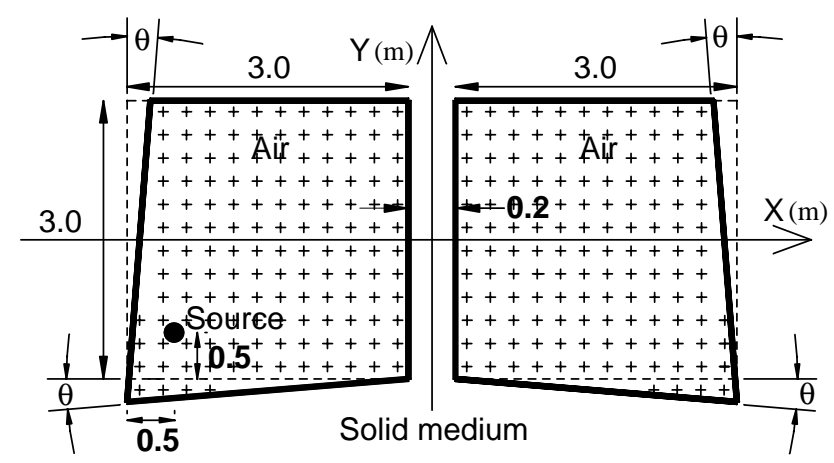

Fig. 1. Geometry of the problem. 
Table 1

Mechanical properties of the elastic and fluid medium

\begin{tabular}{lllll}
\hline Medium & Elastic & & & Fluid \\
\hline Material & Concrete & Ceramic & Cork & Air \\
\hline$\alpha(\mathrm{m} / \mathrm{s})$ & 3499 & 2182 & 430 & 340 \\
$\beta(\mathrm{m} / \mathrm{s})$ & 2245 & 1336 & 282 & - \\
$\rho\left(\mathrm{kg} / \mathrm{m}^{3}\right)$ & 2500 & 1400 & 140 & 1.22 \\
$\eta$ & $4 \times 10^{-3}$ & $2 \times 10^{-3}$ & $15 \times 10^{-3}$ & - \\
\hline
\end{tabular}

$\alpha$ : the compressional wave velocity; $\beta$ : the shear wave velocity; $\rho$ : the density; $\eta$ : the loss factor.

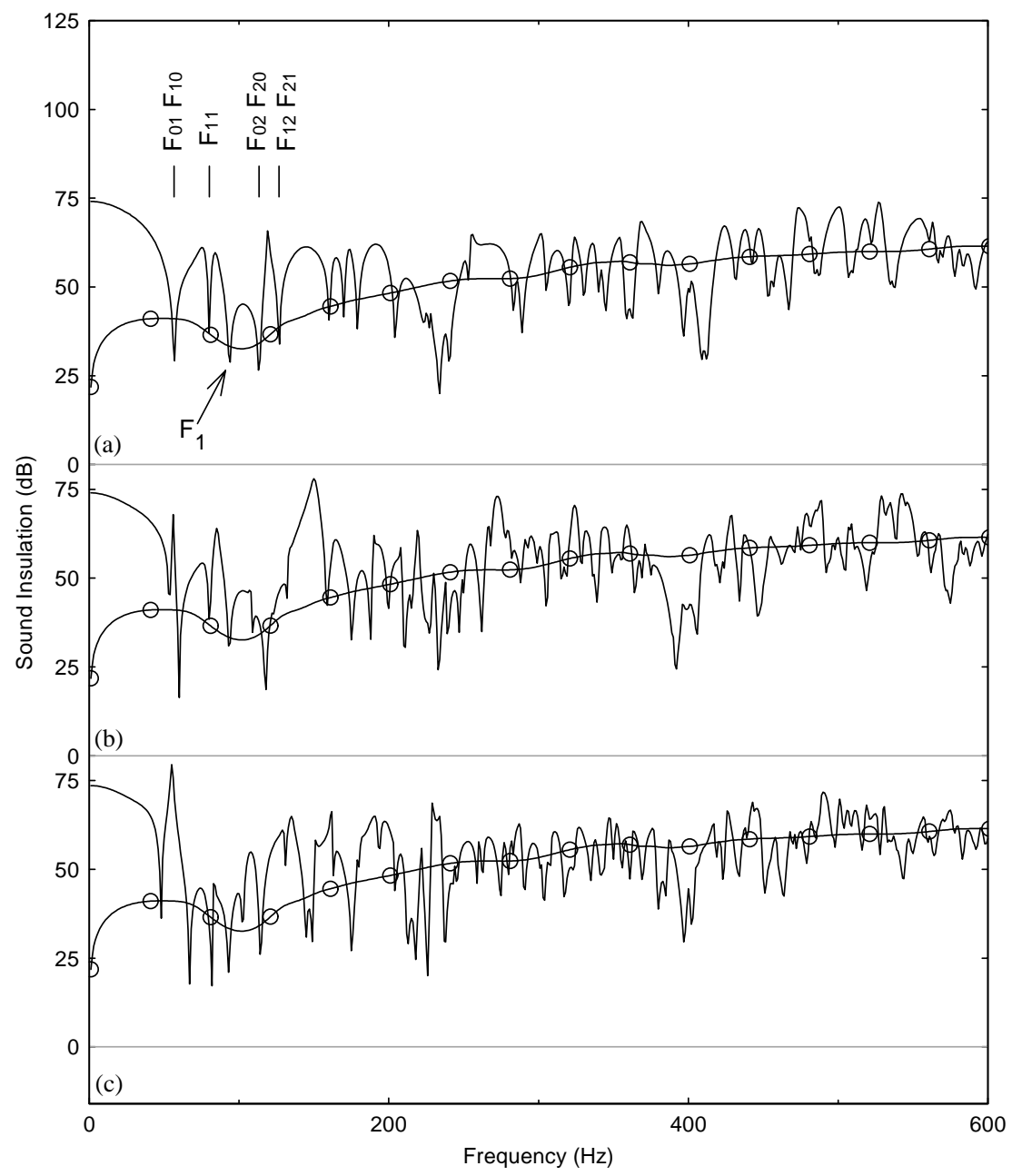

Fig. 2. Average sound insulation provided by the two-room model $(-)$ versus the analytical model $(\odot-)$ when the properties of the concrete is ascribed to the solid medium: (a) $\theta=0^{\circ}$; (b) $\theta=5^{\circ}$; (c) $\theta=15^{\circ}$. 


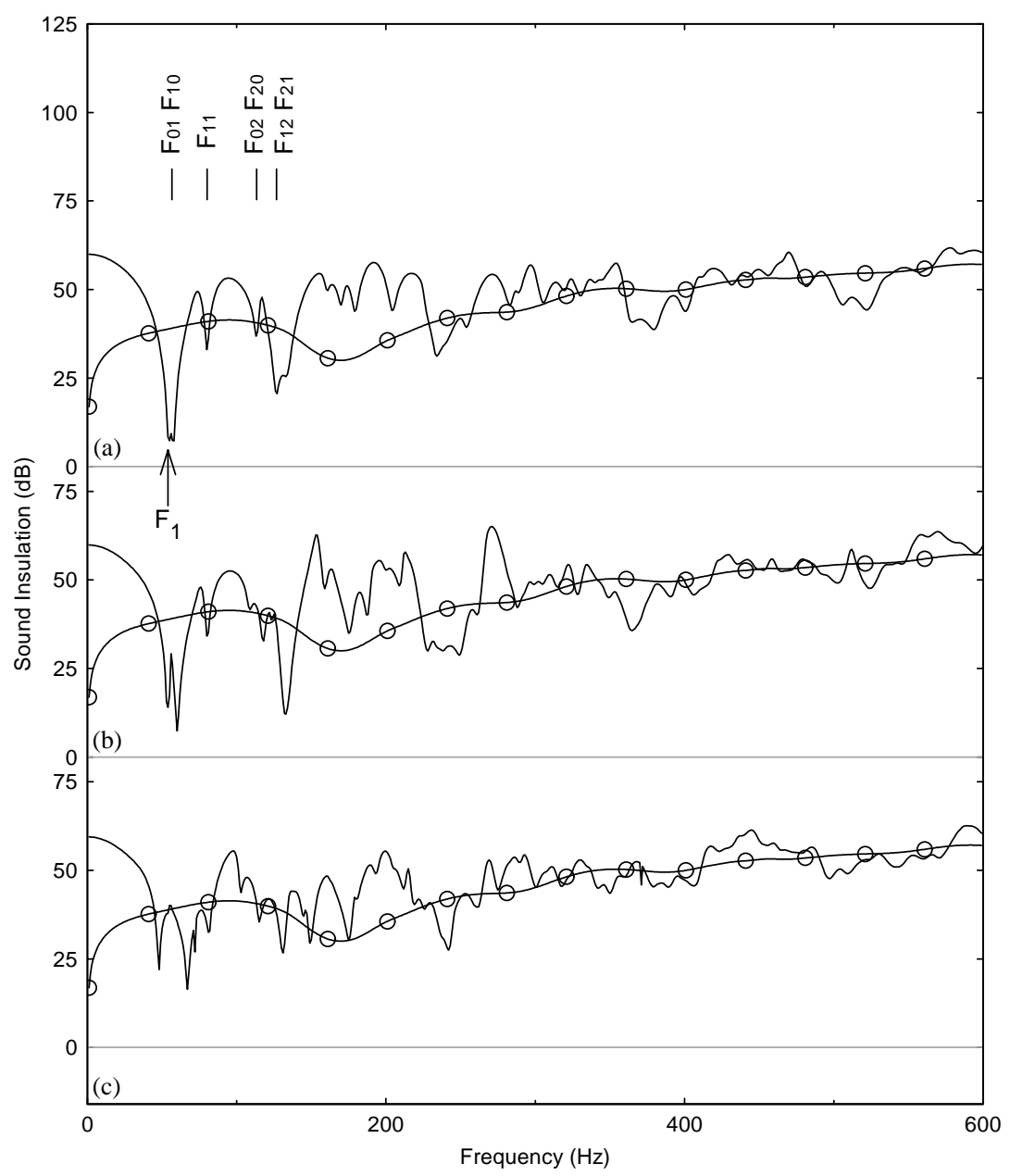

Fig. 3. Average sound insulation provided by the two-room model (- $\longrightarrow$ versus the analytical model $(\odot-)$ ) when the properties of the ceramic is ascribed to the solid medium: (a) $\theta=0^{\circ}$; (b) $\theta=5^{\circ}$; (c) $\theta=15^{\circ}$.

Figs. 2-4 present the average sound insulation provided by the separating wall, computed as the difference between the average sound pressure level registered inside the source and the receiver room, for the three different materials. The analytical solution for a wall of infinite extent is also included in these figures. In this model ("the half-space room") only the separating wall is retained while the floor, ceiling and back walls are removed.

The average sound insulation results obtained when the surrounding material is concrete and $\theta=0^{\circ}$, exhibit pronounced dips (Fig. 2a), as the result of the creation of stationary waves within the rooms and the vibration of the wall in the vicinity of its natural modes of vibration (the first mode is labelled $\mathrm{F}_{1}$ in Figs. 2-4). When $\theta=0^{\circ}$, the stationary waves are created at frequencies given by

$$
F_{q r}=\frac{\alpha_{a}}{2} \sqrt{\left(\frac{q}{3.0}\right)^{2}+\left(\frac{r}{3.0}\right)^{2}}(\mathrm{~Hz})
$$

where $q$ and $r$ are integers $(0,1,2, \ldots)$ (see Fig. 2a). 


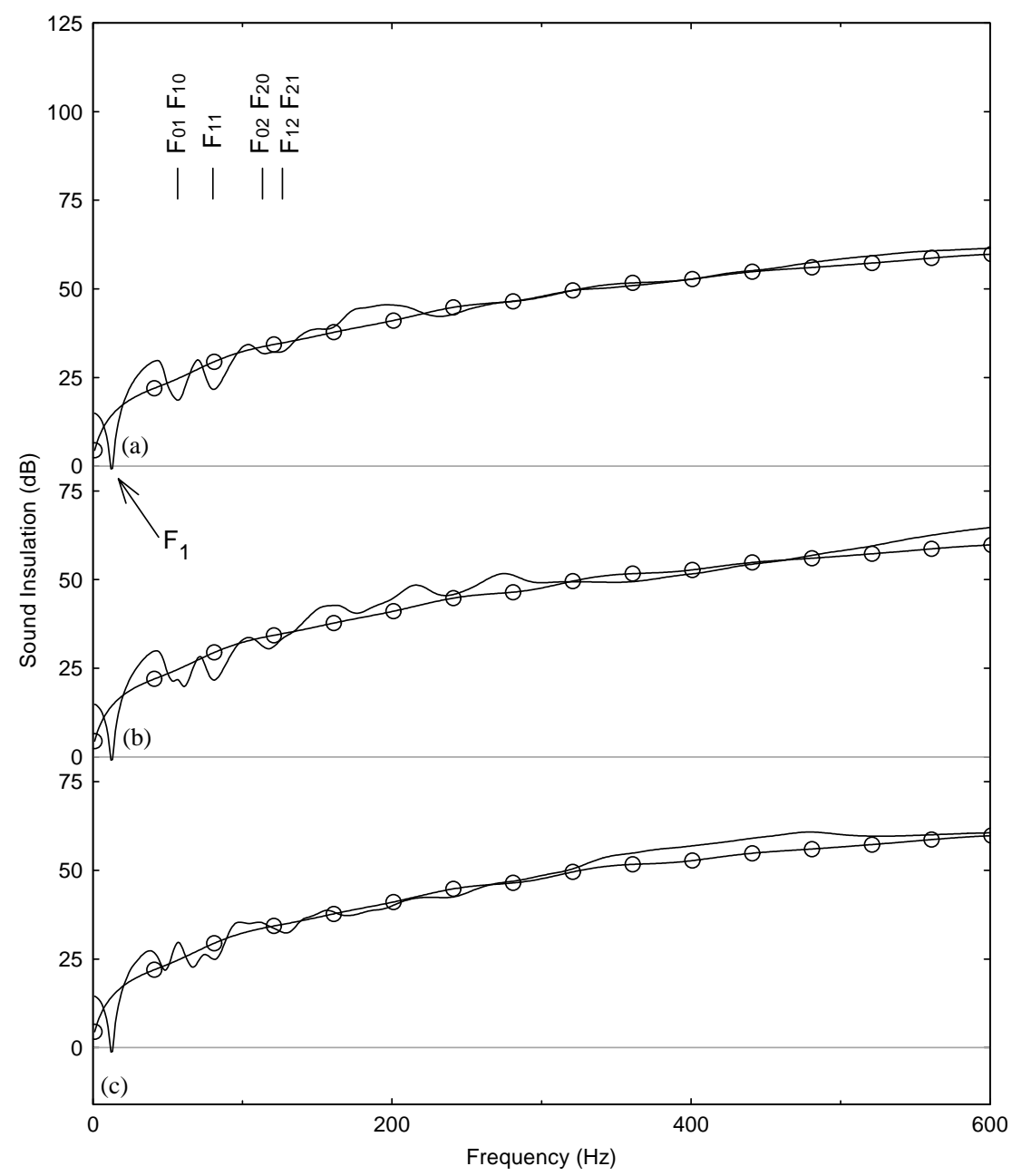

Fig. 4. Average sound insulation provided by the two-room model ( $\_$) versus the analytical model ( $\left.\odot-\odot\right)$ when the properties of the cork is ascribed to the solid medium: (a) $\theta=0^{\circ}$; (b) $\theta=5^{\circ}$; (c) $\theta=15^{\circ}$.

The analytical insulation prediction does not take into account the existence of these dips. However, it intercepts the results provided by the two-room model, except at very low frequencies. There, the acoustic insulation obtained by the two-room model is higher than that predicted by the analytical model.

The analytical solution exhibits a smooth dip of insulation, around $100 \mathrm{~Hz}$, related to the dominant "coincidence effect". Analysis of the results allows verification that the two-room BEM solution tries to follow this trend.

Fig. $2 \mathrm{~b}$ and $\mathrm{c}$ give the sound insulation evaluated when $\theta=5^{\circ}$ and $15^{\circ}$, respectively. The number of insulation dips presented in the two-rooms solution increases, but their amplitude decreases slightly. As expected, the position of the insulation dip associated with the natural vibration of the separating wall does not appear to change as one moves from $\theta=0^{\circ}$ to $5^{\circ}$, and 
then to $\theta=15^{\circ}$. Notice that the "half-space room" results are not affected by $\theta$ and they are thus identical in the three panels of each figure.

Fig. 3 presents the average sound insulation provided when the ceramic properties are used in the model. Analyses of the results allow verification that the dips of the insulation are smoother than before (concrete model), particularly for higher frequencies of excitation. The dips associated with the creation of the stationary wave field inside the rooms and with the vibration of the separating wall are still present. The agreement between the analytical and the two-room model increases, particularly as the frequency and the angle $\theta$ increase. As before, the two-room model does not show a clear dip in the sound insulation associated with the coincidence effect, which agrees with the smooth variation of the analytical solution in the vicinity of the critical frequency.

Fig. 4 plots the acoustic insulation results obtained when the ceramic material is replaced by cork. The insulation curves are much smoother than before (the concrete and ceramic models). The agreement between the analytical and the two-room model solutions further improves, even for the lowest frequencies. The presence of dips associated with the creation of a stationary wave field within the two rooms loses importance particularly as the frequency increases. This can be explained by the fact that the amount of acoustic energy being transmitted to the rooms' boundaries increases the attenuation of the formation of stationary wave field inside the rooms.

\section{Final remarks}

The boundary elements method was applied to assess the influence of the parallelism between the surfaces of two-dimensional acoustic spaces (two-room model), on the sound insulation provided by a single vertical wall, when subjected to a line pressure source. The results obtained were compared with those provided by a wall of infinite extent ("the half-space room") model, which was solved analytically. Both models take into account the fluid-solid interaction.

Different simulations have been performed for different solid materials to assess the importance of the rooms' surface conditions. The ceiling of the acoustic spaces was kept horizontal while the back walls and floor were inclined an angle $\theta\left(\theta=0^{\circ}, 5^{\circ}\right.$ and $\left.15^{\circ}\right)$, in relation to the vertical and horizontal directions, respectively.

The results obtained with the two-room model show that the sound insulation provided by a vertical single wall is highly dependent on the creation of stationary pressure field inside the rooms and on vibration of the separating wall, leading to the existence of pronounced dips of insulation. The break of the parallelism between rooms' surfaces increases the number of dips but only attenuates slightly their amplitude when the solid material is rigid and the frequency of excitation is low. As the frequency increases and as the solid material becomes softer, the attenuation of these dips of insulation appears more visible. This behaviour can be explained by the fact that as the surrounding boundaries become softer more energy is transmitted to the elastic medium, which leads to the weakness of the stationary wave field inside the rooms. As expected, the dips of insulation associated with the natural vibration of the separating wall are the ones less affected by the geometry of the rooms.

The largest differences between the results provided by an infinite extent wall (analytical model) and those obtained when the boundaries of the rooms are modelled, are obtained when the 
cross-sections of the two contiguous acoustic spaces are perfectly rectangular and the spaces are built with a rigid material.

At very low frequencies, the predicted insulation given by the analytical model is lower than that computed by the model, that simulates the boundaries of the rooms. As the frequency increases, the elastic material becomes softer, and the two-room model further loses its parallelism, the differences between the two models decrease.

\section{References}

[1] Lord Rayleigh, The Theory of Sound, Dover, New York, 1945.

[2] Acoustics, Measurement of Sound Insulation in Buildings and of Building Elements, Laboratory Measurements of Airborne Sound Insulation of Building Elements, ISO 140-3, International Standardization Organization, 1978.

[3] Acoustics, Measurement of Sound Insulation in Buildings and of Building Elements, Measurement of Sound Insulation of small Building Elements, ISO 140-10, International Standardization Organization, 1989.

[4] Standard Test Method for Laboratory Measurement of Airborne Sound Transmission Loss of Building Partitions, ASTM E90, American Society for Testing and Materials, 1990.

[5] Acoustics, Measurement of Sound Insulation in Buildings and of Building Elements, Requirements for Laboratories, ISO 140-1, International Standardization Organization, 1990.

[6] J. Mathys, Low frequency noise and acoustical standards, Applied Acoustics 40 (3) (1993) 185-200.

[7] B. Berglund, P. Hassmen, R.F. Soames, Sources and effects of low-frequency noise, Journal of the Acoustical Society of America 99 (5) (1996) 2985-3002.

[8] W. Kropp, A. Pietrzyk, T. Kihlman, On the meaning of the sound reduction index at low frequencies, Acta Acustica 2 (1994) 379-392.

[9] L. Gagliardini, J. Roland, J.L. Guyader, The use of a functional basis to calculate acoustic transmission between rooms, Journal of Sound and Vibration 145 (3) (1991) 457-478.

[10] P. Santos, A. Tadeu, Acoustic insulation provided by a single wall separating two contiguous tunnels via BEM, Journal of Sound and Vibration 257 (5) (2002) 945-965.

[11] A. Tadeu, J. António, Acoustic insulation of single panel walls provided by analytical expressions versus the mass law, Journal of Sound and Vibration 257 (3) (2002) 457-475.

[12] L. Cremer, M. Heckl, E.E. Ungar, Structure-Borne Sound, Springer, Berlin, 1988. 